\title{
TLS-based Interference Alignment Algorithm for Symmetric MIMO Interference Network with CSI Errors
}

\author{
Zhengmin Kong \\ Department of Automation, Wuhan University, Wuhan 430072, Hubei, China \\ E-mail: zmkong@whu.edu.cn
}

\begin{abstract}
The channel state information (CSI) errors undermine the performance of interference $\backslash$ this paper, we model the CSI errors at terminals and propose an IA algorithm based on total least squares (TLS) algorithm for the symmetric MIMO interference system under the assumption of CSI errors. According to the TLS algorithm, the proposed IA algorithm optimizes transmit precoding matrices and minimizes the distortion caused by the imperfect CSI. Numerical results show that the proposed IA algorithm is robust in the symmetric MIMO interference network with CSI errors and has better sum-rate performance than the maximum signal-to-interference-plus-noise ratio (Max SINR) and traditional distributed iterative IA algorithms.
\end{abstract}

Keywords: Interference alignment, Interference networks, Imperfect channel statement information, Transmit precoding

\section{Introduction}

The significance of the interference management has been emphasized in [1]. It is important to design efficient interference management schemes for multiuser wireless networks. Interference alignment (IA) has been proposed as a powerful and promising technique to reduce the interference in multiuser multiple input multiple output (MIMO) interference channels [1-2]. In [1-3], Jafar and his group first characterized the degrees of freedom (DoF) for two-user MIMO interference channels. Since then, IA technique has been applied to structure interfering signals to occupy a reduced-dimensional interference subspace at the receivers, which maximize the sum-rate of system.

Recently, various algorithms for IA and IA-inspired schemes in different scenarios [48] have been proposed. Among them, maximum signal-to-interference-plus-noise ratio (Max SINR) scheme [4] has been regarded as one of the most effective schemes so far, without being proven to be convergent. Some other kinds of iterative algorithms presented in [5-8] are also widely used in IA, while most of these former works rely upon perfect channel state information (CSI). However, the realistic channel estimation (CE) and feedback etc. have errors, and as a result, CSI at the transmitter and receiver is imperfect and has errors in practical systems. Lately, several works on IA schemes with imperfect CSI have focused on this aspect. In [9], the authors investigate an iterative beamforming design algorithm so as to maximize the sum outage rate for MIMO interference networks. A robust lattice alignment method which is designed by using the existing conventional iterative IA algorithm is presented in [10] for quasi-static MIMO interference networks with imperfect CSI. In addition, [11] and [12] investigate the effect of CSI mismatch and develop several adaptive interference alignment with CSI error.

In contrast to previous works above, we use an unbiased parameter estimation approach, namely total least squares (TLS), instead of the conventional method which minimizes the projector distances of the interference subspaces. As a result, the signal estimation problem for the receiver can be easily converted into the problem of solving a linear system of equations. Our goal is to overcome the distortion caused by the imperfect 
CSI and to optimize the sum-rate performance under a given and feasible DoF. For the sake of this, we propose a TLS-based estimation algorithm to mitigate the negative effect from CSI errors on sum-rate performance for the multiuser symmetric MIMO interference network [13]. Compared with the traditional iterative IA algorithm, the proposed TLSbased IA algorithm can improve the sum-rate performance in the scenarios with different variances of error for the direct link estimation and interference links estimations.

The following notations are used in the paper. The superscript ${ }^{T}$, superscript ${ }^{H}$ and superscript ${ }^{*}$ stand respectively for transpose, Hermitian transpose and conjugate. Matrices and vectors are set in bold-face letters. $\mathbf{A}_{i,:}$ denotes the $i$ th row of $\mathbf{A}$, and $\mathbf{A}_{i, i}$ denotes the $i$ th column of $\mathbf{A}$. I is the identity matrix, while $\mathbf{0}$ is the all-zero matrix. $\operatorname{cov}(\mathbf{x})$ denote the covariance matrices of a random vector $\mathbf{x} . E\{\mathbf{A}\}$ and $\operatorname{det}(\mathbf{A})$ denote the expectation and the determinant of $\mathbf{A}$, respectively. $\square^{M \times N}$ is the space of complex $M \times N$ matrices. $\mathrm{CN}(\mathbf{a}, \mathbf{A})$ is the complex Gaussian distribution with mean a and covariance matrix $\mathbf{A}$.

\section{System Model}

We consider a symmetric $K$-user MIMO interference network. Each transmitter is equipped with $M$ transmit antennas to support $d_{k}$ data streams $\left(d_{k} \leq \min (M, N)\right.$ ) for user $k$, where each receiver has $N$ receive antennas, as shown in Figure 1. At receiver $k$, the received signal $\mathbf{y}_{k} \in \square^{N \times 1}$ is given as

$$
\mathbf{y}_{k}=\mathbf{H}_{k k} \mathbf{V}_{k} \mathbf{s}_{k}+\sum_{l \neq k}^{K} \mathbf{H}_{k l} \mathbf{V}_{l} \mathbf{s}_{l}+\mathbf{z}_{k}
$$

where $\boldsymbol{s}_{k}$ represents the data streams from transmitter $k$. The transmitted streams are independent identically distributed (i.i.d.) such that $E\left\{\mathbf{s}_{k} \mathbf{s}_{k}^{H}\right\}=\mathbf{I} . \mathbf{V}_{k} \in \square^{M \times d_{k}}$ is the transmit precoding matrix with unit-norm and linearly independent columns at transmitter $k$, where $d_{k}$ is the DoF to meet feasible IA scheme at transmitter $k$. $\mathbf{H}_{k l} \in \square^{N \times M}$ represents the channel fade coefficients from transmitter $l$ to receiver $k$ for $k, l=1, \ldots, K$, where each element is assumed to obey an independent and identically distributed (i.i.d.) complex Gaussian random process with zero mean and unit variance $\mathrm{CN}(0,1), \mathbf{z}_{k} \in \square^{N \times 1}$ is noise vector at receiver $k$, which is complex-valued AWGN with elements normal distributed as $\mathrm{CN}\left(0, \sigma^{2} \mathbf{I}\right)$.

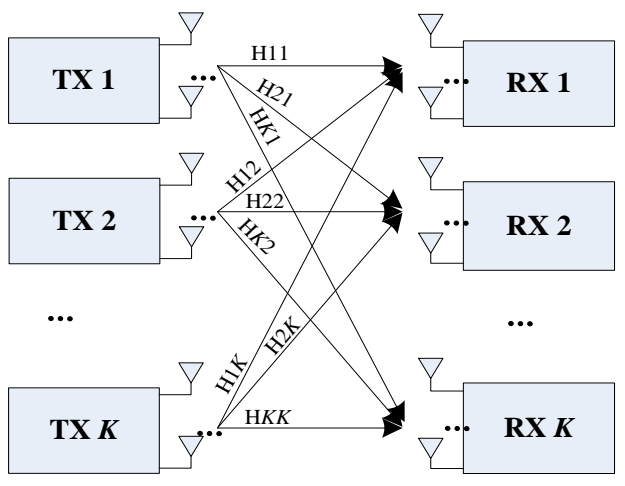

Figure 1. K-user Symmetric MIMO Interference Network Model 
For receive beamforming, the received signal at receiver $k$ in (1) is transformed to

$$
\hat{\mathbf{s}}_{k}=\mathbf{U}_{k}^{H} \mathbf{y}_{k}=\mathbf{U}_{k}^{H} \sum_{l=1}^{K} \mathbf{H}_{k l} \mathbf{V}_{l} \mathbf{s}_{l}+\mathbf{U}_{k}^{H} \mathbf{z}_{k}
$$

where $\mathbf{U}_{k} \in \square^{N \times d_{k}}$ is the receiving filter matrix with unit-norm and linearly independent columns at receiver $k$.

Here, we assume that only imperfect estimation of global CSI is available at each terminal. The feedback channels are assumed to be error-free. The imperfect CSI model can be quantized according to

$$
\mathbf{H}_{k l}=\hat{\mathbf{H}}_{k l}+\tilde{\mathbf{H}}_{k l}, \forall l, k \in\{1,2, \ldots, K\}
$$

where $\hat{\mathbf{H}}_{k l}$ is the estimated channel matrix from transmitter $l$ to receiver $k$, and $\tilde{\mathbf{H}}_{k l}$ is the CSI error matrix between the true and available information.

Due to the CSI errors, (2) can be written as

$$
\hat{\mathbf{s}}_{k}=\mathbf{U}_{k}^{H}\left(\mathbf{H}_{k k}-\tilde{\mathbf{H}}_{k k}\right) \mathbf{V}_{k} \mathbf{s}_{k}+\mathbf{U}_{k}^{H}\left(\sum_{l \neq k}^{K} \mathbf{H}_{k l}-\sum_{l \neq k}^{K} \tilde{\mathbf{H}}_{k l}\right) \mathbf{V}_{l} \mathbf{s}_{l}+\mathbf{U}_{k}^{H} \mathbf{z}_{k}
$$

\section{TLS-based IA Algorithm with CSI Errors}

In this section, the proposed TLS-based IA algorithm will be investigated to solve the IA-aided constrained optimization with CSI errors. This TLS-based IA algorithm is an optimization problem with decision variables, the parameter estimate and the correction.

We assume that $\hat{\mathbf{V}}_{k} \mathbf{s}_{k}$ is the approximation estimation of its true value $(k \in\{1,2, \ldots, K\})$, such that

$$
\hat{\mathbf{s}}_{k}+\mathbf{U}_{k}^{H}\left(-\sum_{l \neq k}^{K} \hat{\mathbf{H}}_{k l} \mathbf{V}_{l} \mathbf{s}_{l}-\mathbf{z}_{k}\right)=\mathbf{U}_{k}^{H} \hat{\mathbf{H}}_{k k} \hat{\mathbf{V}}_{k} \mathbf{s}_{k}
$$

Here, in the beginning of TLS-based optimization, we define a measurement data matrix [14] D with CSI errors at $k$ th receiver

$$
\begin{aligned}
& \mathbf{D} \square\left[\mathbf{U}_{k}^{H} \hat{\mathbf{H}}_{k k} \hat{\mathbf{s}}_{k}-\mathbf{U}_{k}^{H}\left(\sum_{l \neq k}^{K} \hat{\mathbf{H}}_{k l} \hat{\mathbf{V}}_{l} \mathbf{s}_{l}+\mathbf{z}_{k}\right)\right]
\end{aligned}
$$

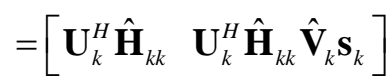

$$
\begin{aligned}
& =\left[\begin{array}{ll}
\mathbf{A} & \mathbf{B}
\end{array}\right]
\end{aligned}
$$

where $\mathbf{A} \square \mathbf{U}_{k}^{H} \hat{\mathbf{H}}_{k k}, \mathbf{B} \square \mathbf{U}_{k}^{H} \hat{\mathbf{H}}_{k k} \mathbf{V}_{k} \mathbf{s}_{k}$. According to (3), the measurement data matrix D can be given by

$$
\begin{aligned}
& \mathbf{D} \square\left[\mathbf{U}_{k}^{H} \mathbf{H}_{k k}-\mathbf{U}_{k}^{H} \tilde{\mathbf{H}}_{k k} \hat{\mathbf{s}}_{k}-\mathbf{U}_{k}^{H}\left(\sum_{l \neq k}^{K} \hat{\mathbf{H}}_{k l} \hat{\mathbf{V}}_{l} \mathbf{s}_{l}+\mathbf{z}_{k}\right)\right] \\
& =\left[\begin{array}{ll}
\mathbf{U}_{k}^{H} \mathbf{H}_{k k} & \hat{\mathbf{s}}_{k}
\end{array}\right]-\left[\begin{array}{ll}
\mathbf{U}_{k}^{H} \tilde{\mathbf{H}}_{k k} & \hat{\mathbf{s}}_{k}-\mathbf{U}_{k}^{H} \hat{\mathbf{H}}_{k k} \hat{\mathbf{V}}_{k} \mathbf{s}_{k}
\end{array}\right] \\
& =\mathbf{D}_{\text {tru }}-\left[\begin{array}{ll}
\tilde{\mathbf{A}} & \tilde{\mathbf{B}}
\end{array}\right] \\
& =\mathbf{D}_{\text {tru }}-\tilde{\mathbf{D}}
\end{aligned}
$$

where $\mathbf{D}_{\text {tru }} \square\left[\mathbf{U}_{k}^{H} \mathbf{H}_{k k} \hat{\mathbf{s}}_{k}\right], \tilde{\mathbf{A}} \square \mathbf{U}_{k}^{H} \tilde{\mathbf{H}}_{k k}, \tilde{\mathbf{B}} \square \hat{\mathbf{s}}_{k}-\mathbf{U}_{k}^{H} \hat{\mathbf{H}}_{k k} \hat{\mathbf{V}}_{k} \mathbf{s}_{k}$ and $\tilde{\mathbf{D}} \square[\tilde{\mathbf{A}} \tilde{\mathbf{B}}]$. $\mathbf{D}_{\text {tru }}$ is the true data matrix $\mathbf{D}, \tilde{\mathbf{D}}$ is the measurement error. And $\tilde{\mathbf{d}}_{i}, i \in\left\{1, \ldots, d_{k}\right\}$ is the $i$ th independent column of $\tilde{\mathbf{D}}^{H}$; it is with row covariance matrices 


$$
\mathbf{W}_{i} \square \operatorname{cov}\left(\tilde{\mathbf{d}}_{i}\right), i \in\left\{1, \ldots, d_{k}\right\}
$$

We define an extended matrix of the approximate estimation $\hat{\mathbf{V}}_{k} \mathbf{s}_{k}$

$$
\hat{\mathbf{X}} \square\left[\begin{array}{c}
\hat{\mathbf{V}}_{k} \mathbf{s}_{k} \\
-\mathbf{I}
\end{array}\right] \square\left[\begin{array}{c}
\mathbf{X} \\
-\mathbf{I}
\end{array}\right], \forall k \in\{1, \ldots, K\}
$$

Provided that the initial data matrix $\mathbf{D}$ is available at the transmitter and the error weights information is $\left\{\mathbf{W}_{i}\right\}_{i=1}^{d_{k}}$, corresponding to each row of data matrix $\mathbf{D}$, which totally depends on the quality of CSI. When the data matrix D is confirmed, we set out to seek for a minimal correction $\Delta \mathbf{D}$ to compensate for the measurement errors $\tilde{\mathbf{D}}$, so that the TLS-based optimization [15] in the presence of a mixture of perfect CSI and CSI errors can be defined as

$$
\begin{gathered}
f(\mathbf{X})=\min _{\Delta \mathbf{d}_{1}, \ldots, \Delta \mathbf{d}_{d_{k}}} \sum_{i=1}^{d_{k}}\left\|\mathbf{W}_{i}^{-\frac{1}{2}} \Delta \mathbf{d}_{i}\right\|_{2}^{2} \\
\text { s.t. } \mathbf{D} \hat{\mathbf{X}}+\Delta \mathbf{D} \hat{\mathbf{X}}=0
\end{gathered}
$$

where $\Delta \mathbf{D}^{H}=\left[\Delta \mathbf{d}_{1}, \Delta \mathbf{d}_{2}, \ldots, \Delta \mathbf{d}_{d_{k}}\right]$. The optimization variables are $\hat{\mathbf{V}}_{k} \mathbf{s}_{k}$ and $\Delta \mathbf{D}$. Let $\left(\hat{\mathbf{V}}_{k, \text { opt }} \mathbf{s}_{k}, \Delta \mathbf{D}_{\text {opt }}\right)$ be an optimal point of the TLS-based optimization problem. Hence, $\hat{\mathbf{V}}_{k, \text { opt }} \mathbf{s}_{k}$ is a TLS estimation of the true value $\mathbf{V}_{k} \mathbf{s}_{k}$ and $\mathbf{D}+\Delta \mathbf{D}_{\text {opt }}$ is an optimal TLS estimation of the true data $\mathbf{D}_{\text {tru }}$.

For a certain $\hat{\mathbf{V}}_{k} \mathbf{s}_{k}, \forall k \in\{1, \ldots, K\}, \hat{\mathbf{X}}$ is fixed and the constraint of (10) can be treated as linear equations to optimize variables $\left\{\Delta \mathbf{d}_{i}\right\}_{i=1}^{d_{k}}$. The set of linear equations in $\left\{\Delta \mathbf{d}_{i}\right\}_{i=1}^{d_{k}}$ can be expressed as

$$
\begin{gathered}
\mathbf{D} \hat{\mathbf{X}}+\Delta \mathbf{D} \hat{\mathbf{X}}=\mathbf{D} \hat{\mathbf{X}}+\left[\begin{array}{c}
\Delta \mathbf{d}_{1}^{H} \hat{\mathbf{X}} \\
\vdots \\
\Delta \mathbf{d}_{d_{k}}^{H} \hat{\mathbf{X}}
\end{array}\right]=0, i=1,2, \ldots, d_{k} \\
\hat{\mathbf{X}}^{H} \Delta \mathbf{d}_{i}=-\left[(\mathbf{D} \hat{\mathbf{X}})_{i,:}\right]^{H}, i=1,2, \ldots, d_{k}
\end{gathered}
$$

Let's define residual matrix $Q(\mathbf{X}) \square \mathbf{D} \hat{\mathbf{X}}$. The $i$ th row of the $Q(\mathbf{X})$ is denoted by $q_{i}^{H}(\mathbf{X})$, namely

$$
Q^{H}(\mathbf{X})=\left[q_{1}(\mathbf{X}), \ldots, q_{d_{k}}(\mathbf{X})\right]
$$

Thus, the constraint of (10) can be treated the same way as

$$
\hat{\mathbf{X}}^{H} \Delta \mathbf{d}_{i}=-q_{i}(\mathbf{X}), i=1,2, \ldots, d_{k}
$$

Then the constrained optimization problem $f(\mathbf{X})$ can be separated according to different $\Delta \mathbf{d}_{i}$. In this way, the constrained minimization problem (10) is transformed to $d_{k}$ independent optimization problems

$$
\begin{gathered}
f(\mathbf{X})=\sum_{i=1}^{d_{k}} f_{i}(\mathbf{X}) \\
f_{i}(\mathbf{X})=\min _{\Delta \mathbf{d}_{i}}\left\|\mathbf{W}_{i}^{-\frac{1}{2}} \Delta \mathbf{d}_{i}\right\|_{2}^{2}, i=1,2, \ldots, d_{k} \\
\text { s.t. } \hat{\mathbf{X}}^{H} \Delta \mathbf{d}_{i}=-q_{i}(\mathbf{X})
\end{gathered}
$$


As a result, the solution of (10) becomes the well-known function [16] as follows.

$$
f(\mathbf{X})=\sum_{i=1}^{m} q_{i}^{H}(\mathbf{X})\left(\hat{\mathbf{X}}^{H} \mathbf{W}_{i} \hat{\mathbf{X}}\right)^{-1} q_{i}(\mathbf{X})=\sum_{i=1}^{m} q_{i}^{H}(\mathbf{X}) R_{i}^{-1}(\mathbf{X}) q_{i}(\mathbf{X})
$$

where $R_{i}(\mathbf{X}) \square \hat{\mathbf{X}}^{H} \mathbf{W}_{i} \hat{\mathbf{X}}$. The optimal solution of $\Delta \mathbf{d}_{i}$ can be derived easily as follows.

$$
\Delta \mathbf{d}_{i, o p t}=-\mathbf{W}_{i} \hat{\mathbf{X}}\left(\hat{\mathbf{X}} \mathbf{W}_{i} \hat{\mathbf{X}}^{H}\right)^{-1} q_{i}(\mathbf{X}), i=1, \ldots, d_{k}
$$

Obviously, the above TLS-based optimization problem is based on an approximation of the first-order optimality condition of (17). And the first-order optimality condition of (17) is

$$
f^{\prime}(\mathbf{X})=0
$$

According to the matrix theory [17], the derivative of $f(\mathbf{X})$ with $\mathbf{X}$ is

$$
\begin{aligned}
f^{\prime}(\mathbf{X})=\sum_{i=1}^{d_{k}} & \left\{R _ { i } ^ { - 1 } ( \mathbf { X } ) q _ { i } ^ { * } ( \mathbf { X } ) \left[\mathbf{a}_{i}^{T}\right.\right. \\
& \left.\left.-q_{i}^{T}(\mathbf{X}) R_{i}^{-1}(\mathbf{X}) \mathbf{X}^{T} \operatorname{cov}\left(\tilde{\mathbf{a}}_{i}^{*}\right)+q_{i}^{T}(\mathbf{X}) R_{i}^{-1}(\mathbf{X}) \operatorname{cov}\left(\tilde{b}_{i}^{*}, \tilde{\mathbf{a}}_{i}^{*}\right)\right]\right\}
\end{aligned}
$$

where $\mathbf{a}_{i}, \tilde{\mathbf{a}}_{i}, b_{i}, \tilde{b}_{i}$ are respectively the $i$ th column of $\mathbf{A}^{H}, \tilde{\mathbf{A}}^{H}, \mathbf{B}^{H}, \tilde{\mathbf{B}}^{H}$. Note that in the TLS-based IA system, $\mathbf{s}_{k}$ and $\hat{\mathbf{X}}$ are vectors, thus the solution of $\mathbf{X}$ is univariate case. In this case, $R_{i}(\mathbf{X})$ is a single component matrix, $q_{i}(\mathbf{X}), b_{i}$ and $\tilde{b}_{i}$ are single component vectors.

Evidently the solution to the TLS-based optimization can be derived from (20) and (19) . In other words, substituting (20) into (19), we rely on the numerical solution method [14] and obtain the $\mathbf{X}_{\mathrm{opt}}$, which approximates to accurate value.

Finally, the estimated transmit precoding matrix $\hat{\mathbf{V}}_{k, \text { opt }}$ can be readily obtained from (9) . Consequently, the series of estimated values $\hat{\mathbf{V}}_{k, \text { opt }}$ are optimized to the true value of the transmit precoding matrix $\mathbf{V}_{k}$ by employing the above TLS-based IA optimization algorithm.

According to the above TLS-based IA optimization algorithm, the sum-rate result for stream $s$ of user $k$ is given by

$$
R_{k, \text { sum }}^{(s)}=\log _{2} \operatorname{det}\left(\mathbf{I}+\hat{\mathbf{Z}}_{k, s}^{-1}\left(\mathbf{H}_{k k}-\tilde{\mathbf{H}}_{k k}\right) \mathbf{V}_{k, s} \mathbf{V}_{k, s}^{H}\left(\mathbf{H}_{k k}-\tilde{\mathbf{H}}_{k k}\right)^{H}\right)
$$

where

$$
\hat{\mathbf{Z}}_{k, s}=\tilde{\mathbf{H}}_{k k} \mathbf{V}_{k, s} \mathbf{V}_{k, s}^{H} \tilde{\mathbf{H}}_{k k}^{H}+\sum_{\substack{t=1 \\ t \neq s}}^{d_{k}} \mathbf{H}_{k k} \mathbf{V}_{k, t} \mathbf{V}_{k, \mathrm{t}}^{H} \mathbf{H}_{k k}^{H}+\sum_{\substack{l=1 \\ l \neq k}}^{K} \sum_{t=1}^{d_{k}} \mathbf{H}_{k l} \mathbf{V}_{k, t} \mathbf{V}_{k, \mathrm{t}}^{H} \mathbf{H}_{k l}^{H}+P_{k, \text { noise }}
$$

$\mathbf{V}_{k, s}$ is the transmit precoding for stream $s$ of user $k, P_{k \text {,noise }}$ is the power of noise at user $k$. The item $\left(\mathbf{H}_{k k}-\tilde{\mathbf{H}}_{k k}\right) \mathbf{V}_{k, s} \mathbf{V}_{k, s}^{H}\left(\mathbf{H}_{k k}-\tilde{\mathbf{H}}_{k k}\right)^{H}$ in (21) is the desired signal power, while the item $\hat{\mathbf{Z}}_{k, s}$ in (21) is interferences plus noise. And the first, second, third and fourth terms of $\hat{\mathbf{Z}}_{k, s}$ in (22) represent the internal perturbation purely by CSI error, interstream interference, other user interference and AWGN, respectively. As a result, all sumrate of $K$-user IA scheme can be derived easily as follows.

$$
R_{\text {sum }}=\sum_{k=1}^{K} \sum_{s=1}^{d_{k}} R_{k, \text { sum }}^{(s)}
$$




\section{Simulation Research}

In this section, simulations are conducted to evaluate the performance of our TLSbased IA algorithm presented in Section III for the symmetric MIMO interference network with imperfect CSI.

Without loss of generality, the CSI errors are assumed to be i.i.d. zero-mean complex Gaussian. The parameters $\alpha$ and $\beta$ indicate the variance of CSI errors for direct link $\tilde{\mathbf{H}}_{k k}$ and interference links $\tilde{\mathbf{H}}_{k l}$ [18], respectively. We first generate $\hat{\mathbf{H}}_{k k}$ and $\hat{\mathbf{H}}_{k l}$ randomly according to a zero-mean complex Gaussian distribution. After $\hat{\mathbf{H}}_{k k}$ and $\hat{\mathbf{H}}_{k l}$ are generated as such, for given $\alpha$ and $\beta$, we generate $\tilde{\mathbf{H}}_{k k}$ and $\tilde{\mathbf{H}}_{k l}$, and then the true channel is determined by (3). In each plot presented in this section, the sum-rate performance is computed via Monte Carlo simulations using 1000 independent channel realizations.

Figure 2 shows the sum-rate performance of different IA algorithms with CSI errors for the case $\alpha=\beta=0.01$, where $K=3$ and $M=N=4$. The theoretical maximum DoF is 6 can be achieved [13], which means each transmitter is with 2 DoF. Hence, this system is symmetrical MIMO interference network. As shown in Figure 2, sum-rates of all algorithms increase as the signal-to-noise ratio (SNR) increases, while they all have the error-floor effect because of the internal perturbation purely by CSI errors and the interstream interferences. It can be observed that the proposed TLS-based IA algorithm outperforms the Max SINR [4] and traditional iterative IA [4] (i.e. CJ08) algorithms over all SNR regions. Here, for the sake of demonstrating the sum-rate performance, the number of iteration for both Max SINR and CJ08 algorithms is fixed to 100 [5]. The Max SINR algorithm shows good performance which is almost comparable to the proposed TLS-based IA algorithm in low SNR region. However, as the SNR increases, the sum rate of TLS-based IA algorithm increases much faster than that of the Max SINR algorithm. 


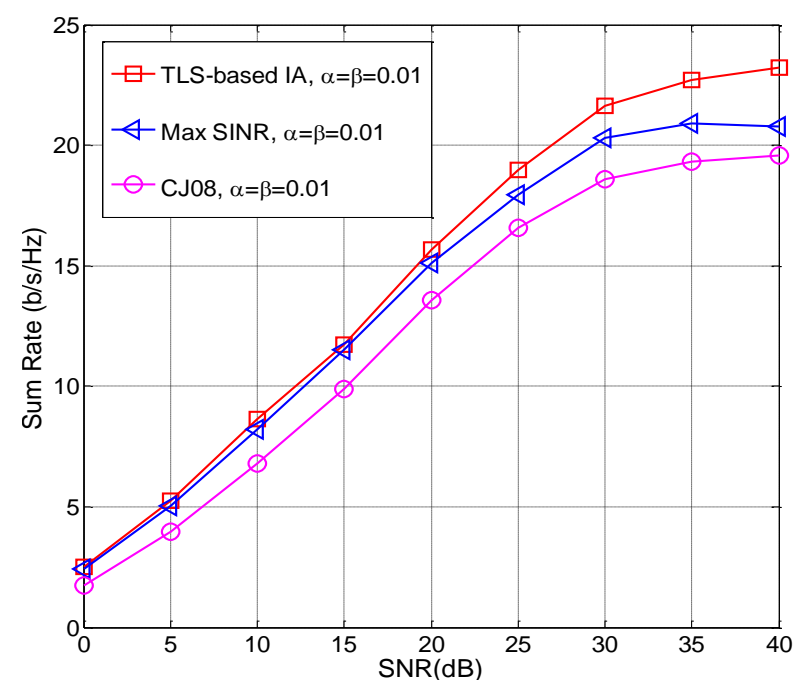

Figure 2. The Sum-Rate Performance of Different IA Algorithms with CSI Errors for $K=3$ and $M=N=4$, and for the Case $\alpha=\beta=0.01$.

Figure 3 describes the sum-rate performance of different IA algorithms with CSI errors for the case $\alpha=\beta=0.1$, where $K=3$ and $M=N=4$. Similar observations can be made in this case, as shown in Figure 2. From both Figure 2 and 3, it can be found that although the sum-rates of all algorithms decrease as the variance of CSI errors increases from 0.01 to 0.1 , the proposed TLSbased IA algorithm always outperforms the other algorithms over all SNR regions in the different scenarios with varied variances of CSI errors. The proposed TLS-based IA algorithm can achieve a better performance than other algorithms even at low SNR. Generally, the proposed TLSbased IA algorithm is proven to have good performance in the scenarios of multiple inter-stream interference.

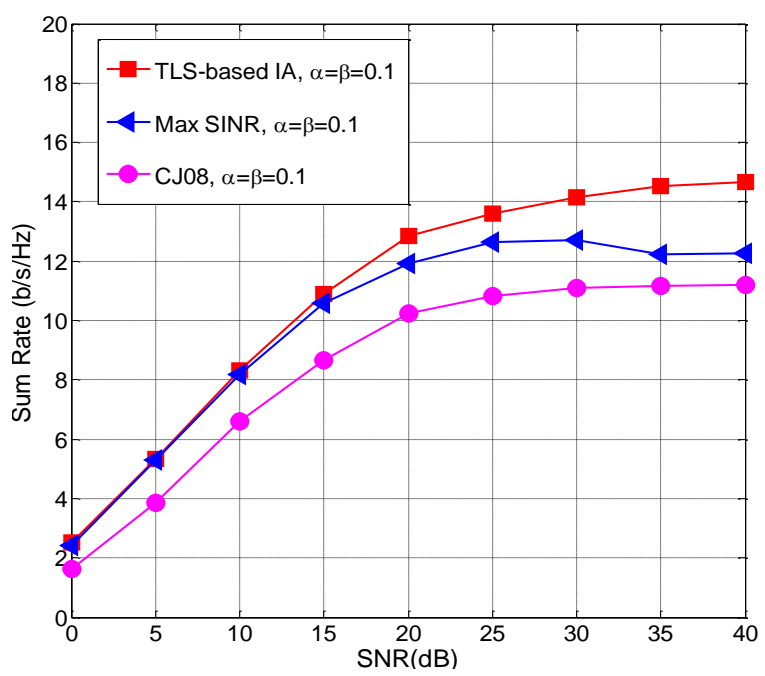

Figure 3. The Sum-Rate Performance of Different IA Algorithms with CSI Errors for $K=3$ and $M=N=4$, and for the Case $\alpha=\beta=0.1$.

Figure 4 portrays the achievable per-stream rate of the proposed TLS-based IA algorithm versus SNR in $K$-user interference channels, where $\alpha=\beta=0.1$. As shown in Figure 4, the achievable per-stream rate monotonically increases as the transmit antennas $M$ and receive antennas $N$ increase, and it saturates in the high SNR regime. Because of the negative effect of inter-user interference, the achievable per-stream rate monotonically decreases as the users increase. It can be also observed that the per-stream rate 
performance of single stream case for each user is much better than that of multiple streams case for each user, since the inter-stream interference has an negative effect on the rate performance.

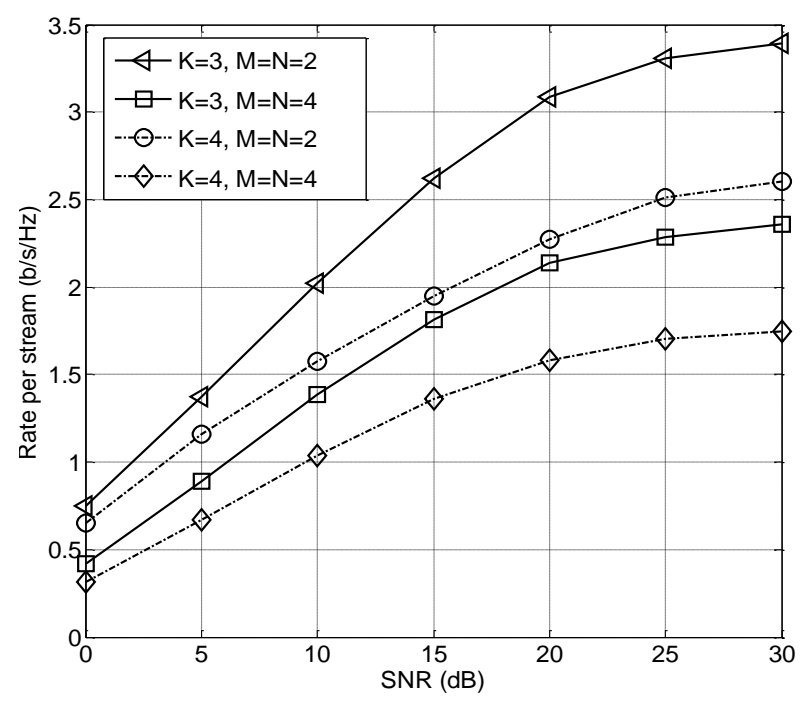

Figure 4. The Achievable Rate per Stream of the Proposed TLS-based IA Algorithm for the Case $\alpha=\beta=0.1$.

\section{Conclusion}

In this paper, we considered the sum-rate performance of different IA algorithms in the scenario of CSI errors. We have proposed an IA-aided algorithm based on TLS error analysis for the transmitted signal estimation optimization with imperfect CSI in symmetric MIMO interference network. The proposed IA algorithm is able to optimize transmit precoding matrices and minimize the distortion caused by imperfect CSI according to the TLS approach. Numerical results showed that the proposed TLS-based IA algorithm can still outperform the several existing IA schemes over all SNR regions in different scenarios of imperfect CSI, although the sum-rate of the TLS-based algorithm decreases as the variance of CSI errors increases.

\section{Acknowledgements}

This work was supported by the National Natural Science Foundation of China (Grant No. 61201168).

\section{References}

[1] S. A. Jafar and S. Shamai, "Degrees of freedom region of the MIMO X channel", IEEE Transactions on Information Theory, vol. 54, (2008), pp. 151-170.

[2] V. R. Cadambe and S. A. Jafar, "Interference alignment and degrees of freedom of the $K$-user interference channel", IEEE Transactions on Information Theory, vol. 54, (2008), pp. 3425-3441.

[3] T. Gou and S. A. Jafar, "Degrees of freedom of the K user MN MIMO interference channel", IEEE Transactions on Information Theory, vol. 56, (2010), pp. 6040-6057.

[4] K. Gomadam and S. A. Jafar, "A distributed numerical approach to interference alignment and applications to wireless interference networks", IEEE Transactions on Information Theory, vol. 57, (2011), pp. 3309-3322.

[5] S. W. Peters and R. W. Heath, "Cooperative algorithms for MIMO interference channels", IEEE Transactions on Vehicular Technology, vol. 60, (2011), pp. 206-218.

[6] $\mathrm{H}$. Yu and Y. Sung, "Least squares approach to joint beam design for interference alignment in multiuser multi-input multi-output interference channels", IEEE Transactions on Signal Processing, vol. 58, (2010), pp. 4960-4966. 
[7] K. Kuchi, "Exploiting spatial interference alignment and opportunistic scheduling in the downlink of interference-limited systems", IEEE Transactions on Vehicular Technology, vol. 63, (2014), pp. 26732686.

[8] J. Yang, R. Song and H. Dong, "Interference alignment scheme for downlink multi-carrier MIMO cellular systems", Journal of Computational Information Systems, vol. 10, (2014), pp. 1053-1064.

[9] J. Park, D. K. Y. Sung and H. V. Poor, "Outage probability and outage-based robust beamforming for MIMO interference channels with imperfect channel state information”, IEEE Transactions on Wireless Communications, vol. 11, (2012), pp. 3561-3573.

[10] H. Huang, V. K. N. Lau and S. Liu, "Robust lattice alignment for K-user mimo interference channels with imperfect channel knowledge", IEEE Transactions on Signal Processing, vol. 59, (2011), pp. 33153325.

[11] B. Xie, Y. Li and A. Nosratinia, "Adaptive interference alignment with CSI uncertainty", IEEE Transactions on Communications, vol. 61, (2013), pp. 792-801.

[12] S. M. Razavi and T. Ratnarajah, "Performance analysis of interference alignment under CSI mismatch", IEEE Transactions on Vehicular Technology, vol. 63, (2014), pp. 4740-4748.

[13] C. M. Yetis, T. Gou, S. A. Jafar and A. H. Kayran, "On feasibility of interference alignment in MIMO interference networks", IEEE Transactions Signal Processing, vol. 58, (2010), pp. 4771-4782.

[14] I. Markovsky, M. Luisa Rastello and S. V. Huffel, "The element-wise weighted total least-squares problem", Computational Statistics and Data Analysis, vol. 50, (2006), pp. 181-209.

[15] A. Kukush and S. V. Huffel, "Consistency of elementwise-weighted total least squares estimator in a multivariate errors-in-variables model AX=B”, Metrika, vol. 59, (2004), pp. 75-97.

[16] P. Sprent, "A generalized least-squares approach to linear functional relationships", Journal of the Royal Statistical Society, Series B (Methodological), vol. 28, (1966), pp. 278-297.

[17] X. Zhang, "Matrix Analysis and Applications", Tsinghua University Press, Beijing, (2004)

[18] H. Farhadi, M. N. Khormuji and M. Skoglund, "Ergodic interference alignment with noisy channel state information", Proceedings of IEEE Internationl Symposium Information Theory (ISIT), (2013); Istanbul, Turkey.

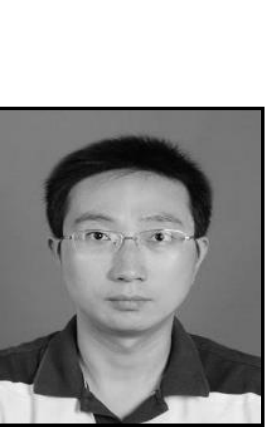

\section{Authors}

Zhengmin Kong, received his B.Eng. degree and Ph.D. degree in 2003 and 2011, respectively, both from the School of Electronic Information and Communications, Huazhong University of Science and Technology, Wuhan, China. From Sep. 2005 to Mar. 2011, he was with the Wuhan National Laboratory for Optoelectronics as a member of research staff and was involved in Beyond-3G (B3G) and UWB system design. He is currently a lecturer in the Department of Automation, Wuhan University. From Mar. 2014 to Mar. 2015, he was with the University of Southampton, UK as an academic visitor and investigated physical layer security and interference management techniques. His current research interests include wireless communications and signal processing, in particular physical layer security and interference management. 
International Journal of Control and Automation Vol.10, No.3 (2017) 